\title{
Generation of an inducible mouse model to reversibly silence Stat3
}

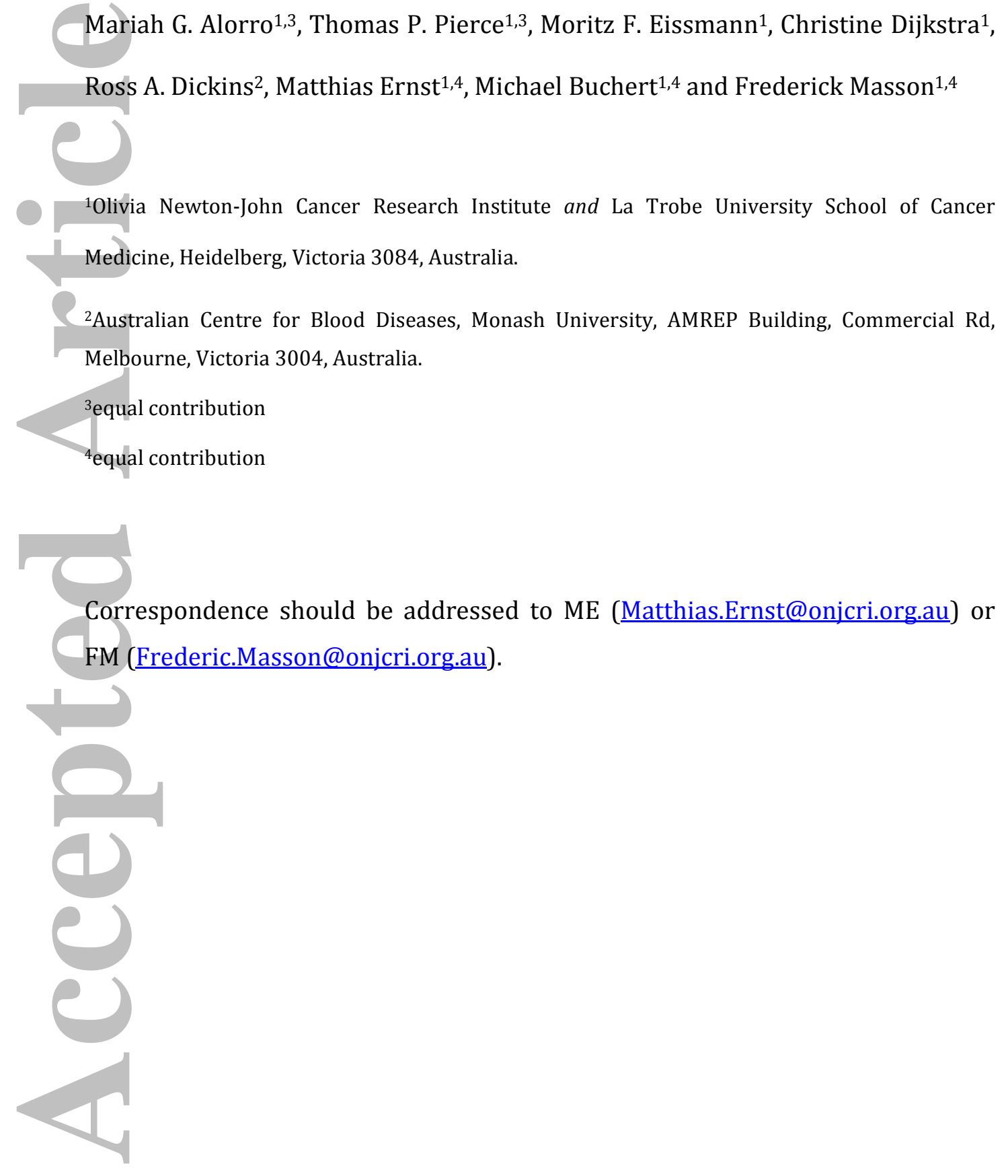

This is the author manuscript accepted for publication and has undergone full peer review but has not been through the copyediting, typesetting, pagination and proofreading process, which may lead to differences between this version and the Version record. Please cite this article as doi:10.1002/ dvg.23023. 


\section{ABSTRACT}

Signal transducer and activator of transcription 3 (Stat3) is a transcription factor that has many essential roles during inflammation, development and cancer. Stat3 is therefore an attractive therapeutic target in many diseases. While current Stat3 knockout mouse models led to a better understanding of the role of Stat3, the irreversible nature of Stat 3 ablation does not model the effects of transient Stat3 therapeutic inhibition, and does not inform on potential dosage effects of Stat3. Using RNAi technology, we have generated a new mouse model allowing the inducible and reversible silencing of Stat3 in vivo, which mirrors the effects of specific Stat3 therapeutic interference. We showed that upon Doxycycline-mediated activation of the Stat3 short-hairpin RNA, Stat3 expression was efficiently reduced by about $80 \%$ in multiple organs and cell types. Moreover, Stat3 reduction was sufficient to reduce tumor burden in a clinically-validated mouse model of gastric cancer. Finally, we demonstrated that Stat3 silencing during embryonic development led to reduced birth rate without leading to complete embryonic lethality, in contrast to full Stat3 ablation. In conclusion, this new mouse model will be invaluable to understand the effects of Stat3 therapeutic interference and Stat3 dosage effects. 


\section{INTRODUCTION}

The signal transducer and activator of transcription 3 (Stat3) is a transcription factor critical for physiological processes in immunity, inflammation, development and metabolism. Meanwhile, aberrant Stat3 activation is often observed in cancers and promotes transcription of genes underpinning a majority of the hallmarks of cancer (Ernst and Putoczki, 2012; Jarnicki et al., 2010; Yu et al., 2009). Consequently, Stat3 and its associated signaling pathways represent attractive targets for anti-cancer therapeutics. This has encouraged the development of STAT3-specific inhibitors including small interfering molecules (BBI608), anti-sense oligonucleotides (AZD9150) or decoy oligonucleotides, which have all entered in clinical trials for the treatment of various forms of cancer (Hong et al., 2015; Li et al., 2015; Sen et al., 2012; Zhang et al., 2016). However, many of these candidate therapeutics require further validation of their clinical potential (Lai et al., 2015).

Much of our current understanding of the role of Stat3 has been derived from studying mice carrying germline or tissue-specific null alleles for Stat3 (Alonzi et al., 2001; Takeda et al., 1998; Takeda et al., 1997). However, these models do not adequately predict the effect of systemic reduction of Stat3 in response to an anti-Stat3 therapy, nor model the consequences of Stat3 re-expression after cessation of anti-Stat3 therapy. To overcome these limitations, we describe here a novel mouse strain that uses RNA interference technology for inducible and reversible Stat3 silencing in vivo and validate this approach in an established mouse model for gastric cancer.

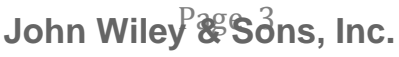




\section{RESULTS AND DISCUSSION}

To generate an inducible mouse model to reversibly silence Stat3, we employed the siRNA scales algorithm (Matveeva et al., 2007) to design short-hairpin RNAs (shRNAs) targeting Stat3, which we cloned into the LMP (LTR-miR30-PuroIRES-eGFP) retroviral expression vector (Dickins et al., 2005). We tested the efficiency of Stat3 silencing in retrovirally transduced immortalized murine embryonic fibroblasts (iMEF), using as a specificity control the LMP-Luc.1309 plasmid, which encodes a shRNA targeting the firefly luciferase. Four of these five shRNA conferred efficient reduction of both Stat3 alpha and beta protein isoforms (Figure 1a). This coincided with reduced transcriptional reporter activity of the 8xAPRE-Luc2 construct, which contains multiple Stat3 binding

sites within the promoter region to control expression of a luciferase reporter. We observed up to $80 \%$ reduction with the shRNA Stat 3.1348 construct, which We selected for further use (Figure 1b).

In order to generate Stat3 shRNA transgenic mice, we introduced the shRNA Stat3.1348 into the ColTGM (ColA1 TRE-eGFP-miR30) plasmid (Premsrirut et al., 2011), which contains a tetracycline (Tet)-responsive promoter (TRE) controlling the expression of shRNA embedded in the miR30 structure and a GFP reporter. We used the resulting plasmid to transfect KH2 ES cells, which contain a "frt-landing pad" in the Col1a1 locus to enable targeted integration in the presence of transiently expressed Flp DNA recombinase (Beard et al., 2006) (Figure 1c). Successfully targeted ES cells clones were then identified through antibiotic selection with hygromycin. Since KH2 ES cells carry a potent Tet-ON transactivator R26-M2rtTA (Premsrirut et al., 2011) to confer inducible 
expression of the shRNA and the associated GFP reporter, we determined the level of Stat3 silencing and GFP expression following Dox treatment (Figure 1d and data not shown). We observed an approximately $50 \%$ reduction of Stat3 protein abundance in 3 out of the 4 clones when grown in the presence of Dox for 5 days. We chose clone D1 for blastocyst injection and subsequent selection of chimeric mice for germline transmission and the establishment of the corresponding inducible Stat3 shRNA transgenic mouse colony (hereafter designated shStat3 mice).

In order to induce high levels of widespread Stat3 shRNA expression upon Dox treatment, the R26-M2rtTA allele was bred out and shStat3 mice were then crossed with CAG-rtTA3 transgenic mice (hereafter designated rtTA mice), which express a sensitive Tet-On transactivator under the control of the strong CAG promoter (Niwa et al., 1991; Premsrirut et al., 2011). Widespread expression of the shRNA-GFP transgene was shown in 2 weeks Dox-treated shStat3;rtTA bitransgenic mice but not in control shStat3 single transgenic mice (Figure 1e).

Next, we determined both the efficiency and reversibility of Stat3 silencing in various organs of shStat3;rtTA bitransgenic mice when compared to control bitransgenic mice harboring the rtTA transgene along with an inducible hairpin transgene against firefly luciferase (shLuc;rtTA mice) (Premsrirut et al., 2011). We treated mice expressing either shRNA for 7 days with Dox-containing chow (7 days On-Dox), or for 7 days with Dox followed by another 7 days with normal chow (7 days-Off-Dox). Within the gastrointestinal tract (i.e. colon, stomach and 
esophagus) of shStat3;rtTA mice Stat3 expression was strongly reduced after 7 days of Dox treatment. However, Stat3 expression was partially recovered after 7 days, but was fully restored 14 days after Dox withdrawal (Figure 2a,b). Although we also observed efficient Dox-dependent reduction of Stat3 in the liver, pancreas, heart, lung and liver, we could not observe any restoration of Stat3 expression within 7 days after Dox withdrawal (Figure 2a), albeit complete recovery of Stat3 expression became apparent in the liver 28 days after Dox withdrawal (Figure 2b). Furthermore, Stat3 expression was not decreased within the spleen suggesting a reduced efficiency of the silencing in immune cells (Figure 2a). Importantly, Dox-dependent reduction of Stat3 did not result in alteration of Stat1 expression confirming the specificity of our short hairpindriven Stat3 silencing system as well as the absence of cross-regulation between Stat3 and Stat1 at steady-state (Supplemental Figure 1). We confirmed coexpression of the shRNA-linked GFP reporter function within the epithelial cells $\left(\right.$ Epcam $\left.^{+} / \mathrm{CD}^{-} 5^{-}\right)$compartment of the stomach, which inversely correlated with the level of Stat3 protein detected in the stomach (Figure 2c). Finally, we verified that shStat3;rtTA mice homozygous or heterozygous for the Stat3-shRNA transgene showed undistinguishable phenotype and similar level of silencing consistent with previous observation that a single copy of the shRNA transgene confers an efficient gene knockdown (Premsrirut et al., 2011) (Supplemental Figure 2).

We then explored whether the heterogeneous level of Stat3 silencing between different organs could reflect differential expression level of the shRNA transgene. Flow cytometric analysis of enzymatically digested stomach and colon 
showed that GFP was homogeneously and highly expressed in Epcam+/CD45epithelial cells (Figure 3a and Supplementary Figure 3). In contrast, endothelial cells (CD31+/Epcam-/CD45-) and mesenchymal cells (Epcam-/CD45-/CD31$/ \mathrm{CD}^{\circ}{ }^{+}$) expressed lower levels of GFP, and within these cell populations the level of GFP was heterogeneous (Figure 3a,c-d).

The lack of Stat3 silencing in the spleen of Dox-treated Stat3-shRNA mice suggested an inefficient shRNA expression in immune cells (Figure 2a,b). Consistent with this hypothesis, GFP reporter expression among B and T cells was low and heterogeneous compared to epithelial cells (Figure 3b-d). The latter observation is likely to reflect differential expression among different subsets of $\mathrm{B}$ and $\mathrm{T}$ cells. Indeed, we found that CD62 $\mathrm{L}^{-} / \mathrm{CD} 44^{+}$effector $\mathrm{CD}^{+} \mathrm{T}$ cells expressed a higher level of the GFP reporter compared to $\mathrm{CD}^{2} \mathrm{~L}^{+} / \mathrm{CD} 44^{-}$naïve and $\mathrm{CD}_{2} 2 \mathrm{~L}^{+} / \mathrm{CD} 44^{+}$central memory $\mathrm{CD}^{+} \mathrm{T}$ cells (Supplementary Figure 4 ). By contrast, GFP expression was considerably higher in myeloid cells (Figure 3b,c) and was uniformly induced in both monocytes and granulocytes upon Dox administration (Figure 3d). Collectively, our data indicated that the Stat3-shRNA and Luc-shRNA are highly and homogeneously expressed in epithelial and myeloid cells, whereas hairpin expression is mosaic in other cell subsets such as mesenchymal cells, and B and T lymphocytes. Therefore we surmise that the Stat3-shRNA mice could serve as a model of choice to study the role of Stat3 in cancers of epithelial origin as well as during normal or malignant myeloid cell development. 
Because Stat3 plays a pivotal role in gastrointestinal cancers (Ernst and Putoczki, 2012), and Stat3 is efficiently silenced in the stomach of Dox-treated shStat3;rtTA mice, we asked whether Stat3 RNA interference technology could reduce gastric cancer development in mice. We therefore exploited homozygous gp130 $1757 \mathrm{~F}$ mice (hereafter gp130 F/F ) as a preclinically-validated mouse model of intestinal-type gastric cancer development. These mice harbor a mutation in the gp130 co-receptor resulting in ligand-dependent Stat3 hyperactivation and spontaneous development of intestinal-type adenomas by 4-5 weeks of age (Jenkins et al., 2005; Thiem et al., 2013). To determine the therapeutic potential of Stat3 reduction in gastric cancer, we generated compound gp130F/F; shStat3;rtTA mice alongside gp130F/F;shLuc;rtTA control mice. In order to control for potential metabolic and/or antibiotic effects of Dox (Moullan et al., 2015), we administered Dox-containing chow for 2 weeks to 18-20 weeks old, tumor-bearing gp130F/F;shStat3;rtTA, gp130F/F;shLuc;rtTA or gp130F/F;shStat3 mice. We observed a significant decrease in tumor burden in gp130F/F;shStat3;rtTA mice when compared to mice from the gp130F/F;shLuc;rtTA or gp130F/F;shStat3 control cohorts (Figure 4a-c). However, tumors from all three cohorts retained similar histopathological features characterized by hyperplastic antrum glands and focal accumulation of inflämmatory cells within the submucosa (Figure 4b). Tumor reduction in gp130F/F;shStat3;rtTA mice coincided with a selective decrease of Stat3 mRNA and protein expression in this strain only (Figure $4 d$, e). Collectively, these data show that the level of Stat3 silencing in $g p 130^{\mathrm{F} / \mathrm{F}}$;shStat3;rtTA mice is sufficient to reduce tumor burden within the stomach.

John Wiley 
Complete Stat3 deficiency leads to early embryonic lethality around E6.5, while Stat3+/- mice develop normally into fertile adults (Takeda et al., 1997). This not only suggests that embryonic and perinatal development may only require less than $50 \%$ of total Stat3 activity, but also that the dosage of Stat3 expression might be key in controlling aspects of early embryonic development. Because, we observed an overall reduction of Stat3 levels by approximately $80 \%$ upon Dox treatment, we hypothesized that shStat3;rtTA mice might provide a model to address this issue genetically. To exclude any potential interference with maternal Stat3 suppression, we set-up matings between C57BL/6 wild-type females and males that harbored the rtTA transgene and were also homozygous for the shStat3 hairpin transgene (shStat3/shStat3;rtTA), and provided Dox food for the entire duration of the pregnancy. We detected widespread GFP expression in shStat3;rtTA newborns (Figure 5a), but we recovered among the postnatal day 1-3 (P1-P3) offspring significantly less shStat3;rtTA bitransgenics than shStat3 single transgenic mice (Figure 5b). However, body weights of live born shStat3;rtTA mice were significantly reduced compared with shStat3 single transgenic littermates (Figure 5c). These observations clearly indicate that shStat3 hairpin activity was sufficient to reduce levels of Stat3 during embryogenesis to below the $50 \%$ required for the development into viable adults. Moreover, our observations are complementary to findings by Darnell and colleagues (Shen et al., 2005), who observed 75\% perinatal lethality in heterozygous Stat3-/S727A mice where the remaining Stat3 allele encodes protein with impaired transcriptional activity . 
Collectively, shStat3;rtTA mice are the first mouse strain allowing a genetic "dial down" of Stat3 expression, in a reversible manner, in a majority of cell types in the adult and during embryonic development. Among other applications, shStat3;rtTA mice are therefore a valuable tool to assess specificity and toxicity of small molecules designed to therapeutically interfere with Stat3 activity.
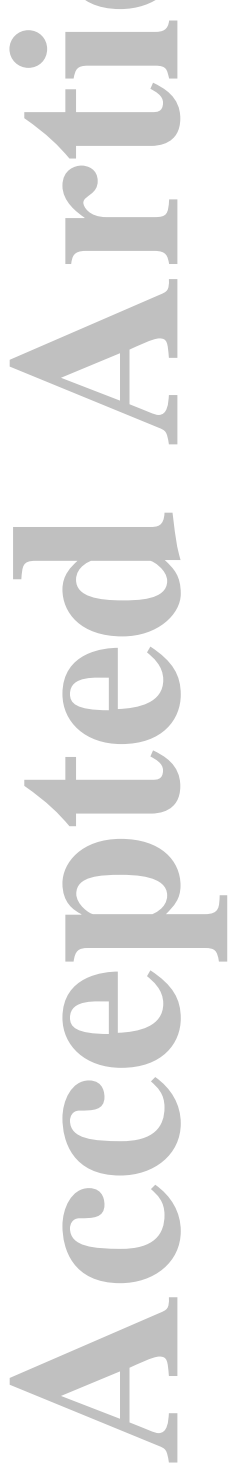

John Wileyagestohs, Inc. 


\section{ACKNOWLEGEMENTS}

The authors thank the Histology and Animal Facility Staff at WEHI and ONJCRI for expert technical assistance. M. Ernst is a Principal Research Fellow of the National Health and Medical Research Council (NHMRC) Australia. This work was supported in part by Project Grant 1069024 and a Program Grant 1092788

from the NHMRC and by funds from the Operational Infrastructure Support
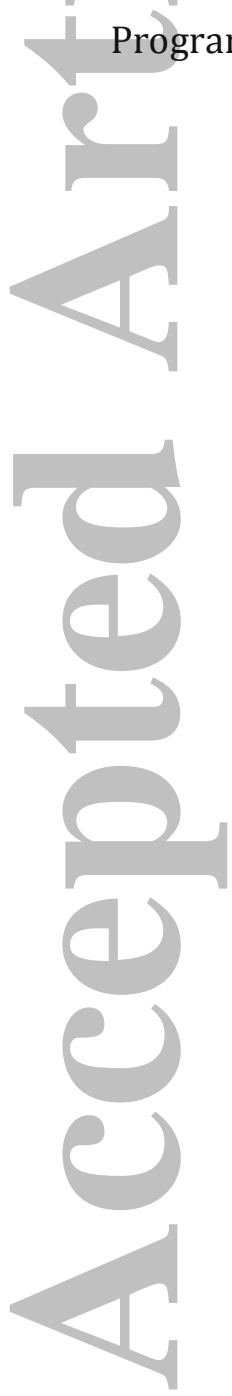

John Wileyagesbrns, Inc.

This article is protected by copyright. All rights reserved. 


\section{MATERIAL AND METHODS}

Mice. The gp130F/F mice (Jenkins et al., 2005; Judd et al., 2004), CAG-rtTA3 and CAG-rtTA3;TRE-Luc.1309 mice have been previously described (Premsrirut et al., 2011), and all mouse strains are in the C57BL/6 background. CAGrtTA3;TRE-Stat3.1348 mice used for experiments were homozygous or heterozygous for the TRE-Stat3.1348 allele (both genotype conferring the same level of Stat3 silencing), while the CAG-rtTA3 allele was kept hemizygous. All mice were maintained under specific pathogenic-free conditions. Aged-matched mice, 7-14 weeks old unless stated otherwise, were used for experiment. For experiments involving analysis of newborn mice, offspring aged between day 1 and day 3 were analyzed. All animal procedures conducted were in accordance with the Animal Ethics Committee of the Olivia Newton-John Cancer Research Institute.

Retroviral transduction and selection. To achieve an average multiplicity of infection (MOI) of 1 virus particle per transduced cell, frozen stocks of retrovirus were thawed at $37^{\circ} \mathrm{C}$, mixed, and serially diluted in growth media. A typical dilution series was as follows: $1 / 10,1 / 30,1 / 100,1 / 300,1 / 1000$, plus nontransduced cells as a control during drug selection. To improve transduction efficiency, hexadimethrine bromide (polybrene, Sigma-Aldrich) was added to virus dilutions to a final concentration of $8 \mu \mathrm{g} / \mathrm{ml}$. Plates were centrifuged at $2700 \mathrm{rpm}(1500 \mathrm{~g})$ for $1 \mathrm{hr}$ at $32^{\circ} \mathrm{C}$. Transduced cells were selected with puromycin dihydrochloride $(2-3 \mu \mathrm{g} / \mathrm{ml})$ (Sigma-Aldrich). 


\section{Cloning shRNA cassettes into ColTGM targeting vector and LMP retroviral}

vector. PCR amplification of the 97mer shRNA sequence was performed from 10ng of oligo template using Phusion ${ }^{\circledR}$ Hot Start Polymerase (Finnzymes) as previously described (Dickins et al., 2007). The 138bp PCR product was digested with XhoI/EcoRI, separated by electrophoresis on a 2\% agarose gel and purified using a QIAquick Gel Extraction Kit (QIAGEN). Purified shRNAs were cloned between the XhoI/EcoRI sites of the retroviral expression vector LMP or the ColTGM targeting vector.

Targeting Constructs and ES cell targeting. The KH2 murine embryonic stem cell line (Beard et al., 2006) was maintained in DMEM Hi Glucose (Gibco) supplemented with 15\% ES-cell qualified FBS (Chemicon), 2mM L- glutamine (Gibco), nucleosides (guanosine $8.5 \mu \mathrm{g} / \mathrm{ml}$, cytidine $7.3 \mu \mathrm{g} / \mathrm{ml}$, thymidine $2.4 \mu \mathrm{g} / \mathrm{ml}$, uridine $7.3 \mu \mathrm{g} / \mathrm{ml}$ and adenosine $8.0 \mu \mathrm{g} / \mathrm{ml}), 0.1 \mathrm{mM} \quad \beta$ mercaptoethanol, 1000U/ml LIF (Chemicon), benzyl penicillin $60 \mu \mathrm{g} / \mathrm{ml}$ and streptomycin sulphate $100 \mu \mathrm{g} / \mathrm{ml}$. Cells were grown on a feeder layer of irradiated G418- and hygromycin- resistant primary mouse embryonic fibroblasts on gelatin-coated tissue culture plates. The cells were electroporated with $50 \mu \mathrm{g}$ of ColTGM plasmid (containing the shRNA of interest) and 25ug of pCAAGS-FLPe-puro. The KH2 cells were subjected to 2 sequential pulses of $400 \mathrm{~V}$, $125 \mu \mathrm{F}$ in a BioRad GenePulser®. Electroporated ES cell were then cultured in complete media in plate containing an established feeder cell layer.

After 40h, surviving clones that have integrated the ColTGM plasmid containing the shRNA cassette were then selected with hygromycin-B $(125 \mu \mathrm{g} / \mathrm{ml})$ for 10 
days. Hygromycin-selected colonies were then picked and transferred to individual wells of a 24-well plate with a pre-established feeder cell layer $\left(1.5 \times 10^{6}\right.$ feeder cells/well). Cells were treated with media containing or not $1 \mu \mathrm{g} / \mathrm{ml}$ doxycycline for 48 hours to induce expression of the shRNA transgene. To confirm integration and function of ColTGM-Stat3.1348 vector in hygromycinresistant KH2 clones, eGFP expression was assessed for each clone using a Nikon Ti-E inverted microscope. The average eGFP fluorescence intensity of all colonies within the field of view was calculated using MetaMorph 7.7.2 (Molecular Devices, USA). For confirmation of Stat3 knock-down by immunoblot, each targeted KH2 cell clone was thawed, expanded and seeded on a feeder cell layer. Clones were treated with or without $1 \mu \mathrm{g} / \mathrm{ml}$ doxycycline for 5 days. The KH2 cells were starved of LIF for 48 hours and then re-stimulated for 5 hours with 1000U/ml LIF prior harvesting.

Generation of the CAG-rtTA;TRE-shStat3 mice. Eight to twelve individual ES cells from the D1 clone were injected into the blastocoel of 3.5 days old blastocysts retrieved from BALB/c mice. Successfully injected blastocysts were subsequently transferred into the uterus of pseudo-pregnant F1 C57BL/6 x 129 or CF1 females. To test for germline transmission, the male chimeras were mated with C57BL/6 females to generate mice F1 heterozygous for the shStat3.1348 allele. Mice were then crossed with the CAG-rtTA3 mice (Premsrirut et al., 2011) to generate the CAG-rtTA;TRE-shStat3 colony.

Doxycycline administration. Doxycycline was incorporated in mouse food pellets (600mg/kg) (Specialty Feeds, Australia). 
Luciferase gene reporter assay. 8xAPRE-Luc2 employs Stat3 binding sequences based on the well-characterized APRE-Luc reporter plasmid (Nakajima et al., 1996). Eight Stat3 binding sites (Wegenka et al., 1993) were inserted into the second-generation firefly luciferase reporter plasmid pGL4.23 (Promega). An oligonucleotide containing the multiple Stat3 binding sequences (TTCTGGGAA) was inserted into the pGL4.23 multiple cloning site between NheI and XhoI, directly upstream of the minimal promoter element. For transfection of iMEFs, Metafectene ${ }^{\circledR}$ PRO (Biontex) transfection reagent was used according to the provider's instructions.

For reporter gene assays, the iMEFs (45,000 cells/well) were plated in 24-well plates. Cells were transfected with 150ng/well of firefly luciferase reporter vector and 150ng/well of pRL-CMV Renilla luciferase vector (Promega) as a transfection control. Triplicate wells were plated for each condition. After overnight incubation, reporter gene activity was assessed using a DualLuciferase ${ }^{\circledR}$ Reporter Assay System (Promega) using automated reagent injection in a Promega GloMax®-96 Microplate Luminometer. Where transfection normalization was employed, reporter gene activity was calculated for each well as a ratio of the firefly and Renilla luciferase signals. Values were then averaged across triplicate wells.

Tissue collection. Mice were euthanized by $\mathrm{CO}_{2}$ prior to tissue collection. Organs were collected and immediately digested for FACS analysis, or snap frozen and stored at $-80^{\circ} \mathrm{C}$ for later protein and RNA extraction. 
Tissue Digestion. Stomach, stomach tumor and colon tissue were enzymatically digested as previously described (Masson et al., 2014).

Flow cytometry. Fluorochrome-conjugated antibodies used for flow cytometric analysis were: Epcam (clone G8.8), CD4 (clone GK1.5), F4/80 (clone BM8), Ly6c (clone HK1.4) all from Ebiosciences; CD8 (clone 53-6.7), B220 (clone RA3-6B2), CD11b (clone M1/70), Ly6g (clone 1A8) all from BD Biosciences; CD90.2 (clone 30-H12), CD31 (clone 390) both from MACS Miltnenyi; CD45.2 (clone S450-152) from the Walter and Eliza Hall institute monoclonal antibody laboratory. SytoxBlue (Invitrogen) was used to exclude dead cells.

Protein extraction and Western blot analysis. Whole tissue lysates were prepared with RIPA lysis buffer supplemented with protease and phosphatase inhibitors (Roche), homogenized using TissueLyser II (Qiagen). Equal amounts (30-45 $\mu \mathrm{g})$ of protein were loaded in $4-12 \%$ Bis-Tris polyacrylamide gel and transferred using the iBlot (Invitrogen). The blots were then probed with antibodies detecting total Stat3 (Cell Signalling, clone 79D7) and Gapdh (Cell Signalling, clone G945). Blots were developed using Pierce ECL substrate (Thermo Fisher Scientific) and visualized using the ChemiDoc MP system (BioRad).

RNA isolation and quantitative RT-PCR. RNA was extracted from frozen tumor tissue using Trizol RNA isolation (Invitrogen). $2 \mu \mathrm{g}$ of resulting RNA was then converted into cDNA using the High-Capacity cDNA Reverse Transcription Kit (Applied Biosystems). Quantitative RT-PCR analyses were performed in 
triplicates samples using SensiMix ${ }^{\mathrm{TM}}$ SYBR reagent (Bioline) on the $\mathrm{Viia}^{\mathrm{TM}} 7$ RealTime PCR System (Life technologies). Relative Stat3 gene expression was calculated using the delta Ct method normalized to Gapdh gene expression.

Primers used:

Stat3 Fw: AGAACCTCCAGGACGACTTTG

Rv: TCACAATGCTTCTCCGCATCT

Gapdh Fw: CACTGAGCATCTCCCTCACA

Rv: GTGGGTGCAGCGAACTTTAT

IVIS imaging. Mice were euthanized and analyzed for GFP fluorescence $(430 \mathrm{~nm}$ and $465 \mathrm{~nm}$ excitation filters) using the Vivo Vision Lumina In Vivo Imaging system (IVIS, Xenogen).

Statistical analysis. Unless stated otherwise, all statistical analysis were performed with the Mann-Whitney U test using Prism 6 software (GraphPad Software).

Declaration. The CAG-rtTA;TRE-shStat3 strain will be made available to the research community in accordance to relevant Material Transfer Agreements.

John Wileyagestrns, Inc.

This article is protected by copyright. All rights reserved. 


\section{REFERENCES}

Alonzi, T., Maritano, D., Gorgoni, B., Rizzuto, G., Libert, C., and Poli, V. (2001). Essential role of STAT3 in the control of the acute-phase response as revealed by inducible gene inactivation [correction of activation] in the liver. Mol Cell Biol21, 1621-1632.

Beard, C., Hochedlinger, K., Plath, K., Wutz, A., and Jaenisch, R. (2006). Efficient method to generate single-copy transgenic mice by site-specific integration in embryonic stem cells. Genesis 44, 23-28.

Dickins, R.A., Hemann, M.T., Zilfou, J.T., Simpson, D.R., Ibarra, I., Hannon, G.J., and Lowe, S.W. (2005). Probing tumor phenotypes using stable and regulated synthetic microRNA precursors. Nature Genetics 37, 1289-1295.

Dickins, R.A., McJunkin, K., Hernando, E., Premsrirut, P.K., Krizhanovsky, V., Burgess, D.J., Kim, S.Y., Cordon-Cardo, C., Zender, L., Hannon, G.J., and Lowe, S.W. (2007). Tissue-specific and reversible RNA interference in transgenic mice. Nature Genetics 39, 914-921.

Ernst, M., and Putoczki, T.L. (2012). Stat3: linking inflammation to (gastrointestinal) tumourigenesis. Clinical and Experimental Pharmacology \& Physiology 39, 711-718.

Hong, D., Kurzrock, R., Kim, Y., Woessner, R., Younes, A., Nemunaitis, J., Fowler, N., Zhou, T., Schmidt, J., Jo, M., et al. (2015). AZD9150, a next-generation antisense oligonucleotide inhibitor of STAT3 with early evidence of clinical activity in lymphoma and lung cancer. Science Translational Medicine 7, 314ra185.

Jarnicki, A., Putoczki, T., and Ernst, M. (2010). Stat3: linking inflammation to epithelial cancer - more than a "gut" feeling? Cell Division 5, 14.

Jenkins, B.J., Grail, D., Nheu, T., Najdovska, M., Wang, B., Waring, P., Inglese, M., McLoughlin, R.M., Jones, S.A., Topley, N., et al. (2005). Hyperactivation of Stat3 in 
gp130 mutant mice promotes gastric hyperproliferation and desensitizes TGFbeta signaling. Nature Medicine 11, 845-852.

Lai, P.S., Rosa, D.A., Magdy Ali, A., Gomez-Biagi, R.F., Ball, D.P., Shouksmith, A.E., and Gunning, P.T. (2015). A STAT inhibitor patent review: progress since 2011. Expert Opin Ther Pat 25, 1397-1421.

Li, Y., Rogoff, H.A., Keates, S., Gao, Y., Murikipudi, S., Mikule, K., Leggett, D., Li, W., Pardee, A.B., and Li, C.J. (2015). Suppression of cancer relapse and metastasis by inhibiting cancer stemness. Proc Natl Acad Sci U S A 112, 1839-1844.

Masson, F., Ghisi, M., Groom, J.R., Kallies, A., Seillet, C., Johnstone, R.W., Nutt, S.L., and Belz, G.T. (2014). Id2 represses E2A-mediated activation of IL-10 expression in T cells. Blood 123, 3420-3428.

Matveeva, O., Nechipurenko, Y., Rossi, L., Moore, B., Saetrom, P., Ogurtsov, A.Y., Atkins, J.F., and Shabalina, S.A. (2007). Comparison of approaches for rational siRNA design leading to a new efficient and transparent method. Nucleic Acids Research 35, e63.

Moullan, N., Mouchiroud, L., Wang, X., Ryu, D., Williams, E.G., Mottis, A., Jovaisaite, V., Frochaux, M.V., Quiros, P.M., Deplancke, B., Houtkooper, R.H. and Auwerx, J. (2016). Tetracyclines Disturb Mitochondrial Function across Eukaryotic Models: A Call for Caution in Biomedical Research. Cell Reports 10,1681-1691

Nakajima, K., Yamanaka, Y., Nakae, K., Kojima, H., Ichiba, M., Kiuchi, N., Kitaoka, T., Fukada, T., Hibi, M., and Hirano, T. (1996). A central role for Stat3 in IL-6induced regulation of growth and differentiation in M1 leukemia cells. EMBO J $15,3651-3658$.

Niwa, H., Yamamura, K., and Miyazaki, J. (1991). Efficient selection for highexpression transfectants with a novel eukaryotic vector. Gene 108, 193-199.

John Wileyasestons, Inc.

This article is protected by copyright. All rights reserved. 
Premsrirut, P.K., Dow, L.E., Kim, S.Y., Camiolo, M., Malone, C.D., Miething, C., Scuoppo, C., Zuber, J., Dickins, R.A., Kogan, S.C., et al. (2011). A rapid and scalable system for studying gene function in mice using conditional RNA interference. Cell 145, 145-158.

Sen, M., Thomas, S.M., Kim, S., Yeh, J.I., Ferris, R.L., Johnson, J.T., Duvvuri, U., Lee, J., Sahu, N., Joyce, S., et al. (2012). First-in-human trial of a STAT3 decoy oligonucleotide in head and neck tumors: implications for cancer therapy. Cancer Discovery 2, 694-705.

Shen, Y., La Perle, K.M., Levy, D.E., and Darnell, J.E., Jr. (2005). Reduced STAT3 activity in mice mimics clinical disease syndromes. Biochem Biophys Res Commun 330, 305-309.

Takeda, K., Kaisho, T., Yoshida, N., Takeda, J., Kishimoto, T., and Akira, S. (1998).

Stat3 activation is responsible for IL-6-dependent $\mathrm{T}$ cell proliferation through preventing apoptosis: generation and characterization of T cell-specific Stat3deficient mice. J Immunol 161, 4652-4660.

Takeda, K., Noguchi, K., Shi, W., Tanaka, T., Matsumoto, M., Yoshida, N., Kishimoto, T., and Akira, S. (1997). Targeted disruption of the mouse Stat3 gene leads to early embryonic lethality. Proc Natl Acad Sci U S A 94, 3801-3804.

Thiem, S., Pierce, T.P., Palmieri, M., Putoczki, T.L., Buchert, M., Preaudet, A., Farid, R.O., Love, C., Catimel, B., Lei, Z., et al. (2013). mTORC1 inhibition restricts inflammation-associated gastrointestinal tumorigenesis in mice. Journal of Clinical Investigation 123, 767-780.

Wegenka, U.M., Buschmann, J., Lutticken, C., Heinrich, P.C., and Horn, F. (1993). Acute-phase response factor, a nuclear factor binding to acute-phase response elements, is rapidly activated by interleukin- 6 at the posttranslational level. Mol Cell Biol 13, 276-288.

John Wileyagesôns, Inc. 
Yu, H., Pardoll, D., and Jove, R. (2009). STATs in cancer inflammation and immunity: a leading role for STAT3. Nature Reviews. Cancer 9, 798-809.

Zhang, Y., Jin, Z., Zhou, H., Ou, X., Xu, Y., Li, H., Liu, C., and Li, B. (2016). Suppression of prostate cancer progression by cancer cell stemness inhibitor napabucasin. Cancer Medicine 5, 1251-1258.

John Wileyagesơns, Inc. 


\section{FIGURE LEGENDS}

Figure 1. Generation of an inducible Stat3 short-hairpin RNA mouse model.

(a) Western blot of cell lysate from iMEF cells transfected with LMP retroviral vectors expressing candidate Stat3 targeting shRNAs. Data are representative of 2 independent experiments.

(b) Reporter gene assay showing 8xAPRE-Luc2 reporter signal as a measure of efficacy of the shRNA mediated Stat3 activity suppression. Data shows mean \pm SEM from technical triplicates.

(c) Schematic of the site-specific integration of the ColTGM vector that contains the shRNA into the genome of KH2 embryonic stem cells (ColA1 TRE-eGFP-miR30). (1) A "landing pad" is knocked in on the 3'UTR site of the ColA1 locus of the KH2 embryonic stem cells (Beard et al., 2006). The landing pad consists of a P PGK and a neomycin resistant gene $\left(\mathrm{Neo}^{\mathrm{R}}\right)$ cassette that is flanked by frt sequences. Downstream to this cassette is an ATG-less hygromycin resistance cassette (Hygro $^{\mathrm{R}}$ ). (2) The ColTGM vector comprises a strong Dox-responsive promoter $\left(\mathrm{P}_{\mathrm{TRE}}\right)$ to drive the expression of the eGFP and shRNA located downsteam to the promoter and ATG translational start codon, followed by an frt site to allow site specific recombination. (3) FLPe mediated recombination leads to excision of the PGK-Neo ${ }^{R}$ cassette and site-specific recombination of the ColTGM vector in the ColA1 locus resulting in restoration of the hygromycin resistance.

(d) Western blot of four KH2 ES cell colonies targeted with the ColTGM Stat3.1348 plasmid and treated with or without Dox as indicated. Data are representative of 2 independent experiments.

(e) Representative IVIS derived images showing GFP expression in 2 weeks Dox treated shStat3 (left) and shStat3;rtTA (right) mice. 
Figure 2. The level of shRNA-mediated Stat3 suppression and restoration is variable between different tissues.

(a) Western blot analysis of total Stat3 and Gapdh expression in whole tissue lysate from the indicated organs of shStat3;rtTA and control shLuc;rtTA mice, either left untreated or treated for 7 days with Dox and analyzed directly after treatment (ON-Dox) or after 7 days of Dox withdrawal (OFF-Dox), one lane representative of one experiment while stomach, liver colon and spleen blots are representative of 3 experiments.

(b, c) The shStat3;rtTA and control shLuc;rtTA mice were treated 7 days with Dox (ON-Dox) and were analyzed at $0,7,14$ or 28 days after Dox withdrawal (OFF-Dox).

(b) Western blot analysis of total Stat3 and Gapdh expression in whole tissue lysate from stomach, liver, colon and spleen of Dox-treated shStat3;rtTA and control shLuc;rtTA mice, each lane represents one mouse. The time-points untreated, 7 days ON-Dox and 7 days OFF-Dox are representative of three independent experiments (each with $n=2-3$ mice), while the 14 days OFF-Dox are representative of 2 experiments (each with $n=2$ mice), and 28 days OFF-Dox are representative of one experiment ( $\mathrm{n}=2$ mice).

(c) Representative flow cytometric analysis of GFP expression on gated epithelial cells $\left(\right.$ Epcam $^{+} / \mathrm{CD}_{45}$ ) from enzymatically digested stomach tissue, each plot represents one mouse. Plots are representative of one experiment with 2 mice per time point. 
Figure 3. Heterogeneity of the shRNA expression across hematopoietic and non-hematopoietic cells.

shStat3, shStat3;rtTA, and shLuc;rtTA mice were treated for 7 days with Dox.

(a) Representative FACS plots showing gating strategies and GFP expression in stomach epithelial (CD45-/Epcam ${ }^{+}$), mesenchymal (CD45/Epcam-/CD31/CD90.2 $2^{+}$) and endothelial cells (CD45-/Epcam-/CD31+/CD90.2-) of shStat3;rtTA (green line) and shStat3 (grey line) mice, following 1 week of Dox treatment.

(b) Representative FACS plots showing gating strategies and GFP expression in the splenic CD4 T cells (CD4+/CD8-), CD8 T cells (CD4-/CD8+), B cells (CD4-/CD8/B220 $\left.{ }^{+}\right)$monocytes $\left(\mathrm{CD}_{\left.11 \mathrm{~b}^{+} / \mathrm{Ly} \mathrm{C}^{+} / \mathrm{Ly} 6 \mathrm{G}^{-}\right)}\right)$and granulocytes $\left(\mathrm{CD}_{11 \mathrm{~b}^{+}} / \mathrm{Ly} 6 \mathrm{C}^{\text {low }} / \mathrm{Ly}_{6 \mathrm{G}}{ }^{+}\right.$) from shStat3;rtTA (green line) and shStat3 (grey line) mice following 1 week of Dox treatment.

(c) Bar graph shows the mean \pm SEM of the GFP geometric mean fluorescence intensity (GMFI) in shStat3;rtTA or shLuc;rtTA mice normalized to GFP GMFI in shStat3 mice within each of the indicated cell populations. Statistical differences were assessed with Mann-Whitney U statistical test.

(d) Bar graph shows the mean \pm SEM percentage of GFP positive cells within each indicated population. Data are representative $(a, b)$ or cumulative $(c, d)$ of $\mathrm{n}=4$ mice per genotype pooled from 2 independent experiments.

John Wileyagesóns, Inc.

This article is protected by copyright. All rights reserved. 
Figure 4. Stat3 silencing reduced tumor burden in a model of gastric tumorigenesis.

(a) Representative images of (18-20 weeks old) gp130F/F;shLuc;rtTAand gp130F/F; shStat3;rtTA mice after 2 weeks of Dox treatment.

(b) Hematoxylin \& Eosin-stained cross sections through stomachs from 2 weeks

Dox-treated (18-20 weeks old) gp130/F;shLuc;rtTA and gp130F/F;shStat3;rtTA mice. Images are representative of 6 gp $130^{\mathrm{F} / \mathrm{F}}$;shLuc;rtTA mice from 4 independent experiments and 16 gp130 $16 / \mathrm{F} ; \operatorname{shStat} 3 ;$ rtTA mice from 5 independent experiments.

(c) Tumor mass in 2 weeks Dox-treated gp130 F/F;shStat3;rtTA mice compared to gp130F/F;shLuc;rtTA and gp130F/F;shStat3 control mice, where one symbol represents one mouse. Data shows mean \pm SEM of the total tumor mass, with all shLuc;rtTA mice pooled from 3 independent experiments and shStat;rtTA and shStat3 mice pooled from 6 independent experiments.

(d) Quantitative RT-PCR analysis of Stat3 expression in gastric tumor tissue collected from 2 weeks Dox-treated gp130F/F;shStat3;rtTA compared to the gp130F/F;shLuc;rtTA and gp130F/F;shStat3 controls. Values are normalized to Gapdh expression, and bar indicates mean \pm SEM of Stat3 normalized expression. (e) Western blot analysis of total Stat3 and Gapdh in whole gastric tumor tissue of 2 weeks Dox-treated shStat3;rtTA. Samples are from 2 (shStat3), 3 (shLuc;rtTA), and 6 (shStat3;rtTA) independent experiments, each with $n=2-3$ mice.

The shLuc;rtTA mice are pooled from 3 independent experiments and the shStat3 and shStat3;rtTA are pooled from 6 independent experiments. Each 
symbol represents one mouse (c,d). Statistical differences were assessed with Mann-Whitney U statistical test, ${ }^{*} \mathrm{p}<0.05 ;{ }^{* *} \mathrm{p}<0.01$.
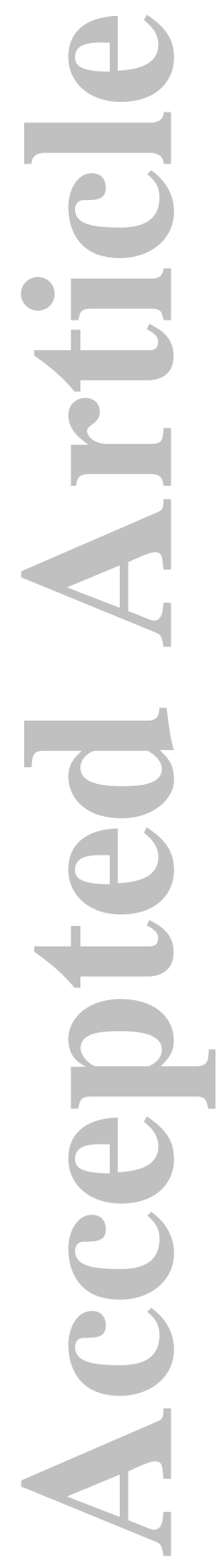

John Wileyagesóns, Inc.

This article is protected by copyright. All rights reserved. 


\section{Figure 5. Stat3 inhibition during embryonic development results in} decrease survival.

Wild-type females were mated with shStat3/shStat3;rtTA males and Dox was given throughout mating and pregnancy.

(a) Representative IVIS image showing GFP expression in 1 day old offspring.

(b) Table shows expected and observed number of offspring, and the Chi-square test value and its associated p-value, assessing statistical significant difference between observed and expected frequency of each genotype.

(c) Bar graph shows mean \pm SEM normalized total body weight of day 1-3 old offspring (normalized to the average body weight of shStat3 offspring) of shStat3;rtTA and shStat3 newborn mice where one symbol represents one mouse. Statistical differences were assessed with Mann-Whitney U statistical test.

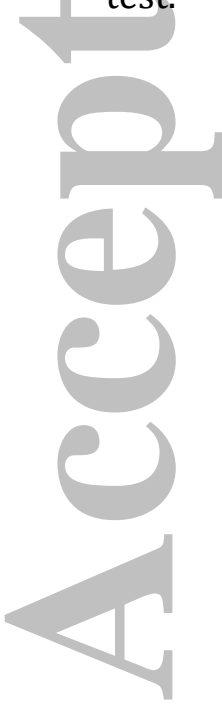

John Wileyases27ns, Inc.

This article is protected by copyright. All rights reserved. 
a

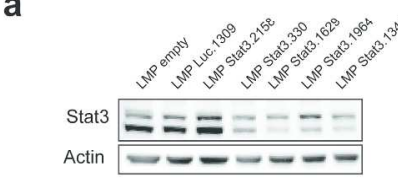

c

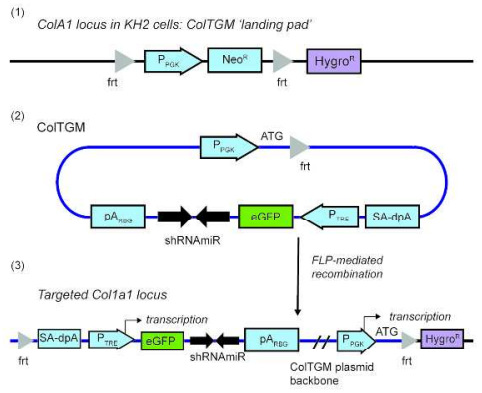

b

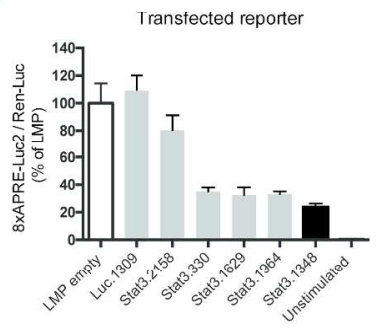

d

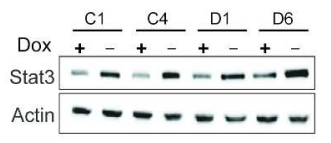

e

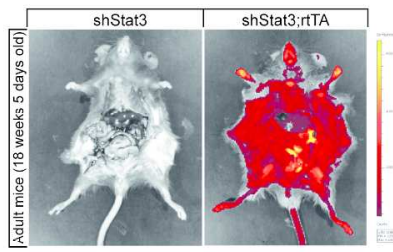

Figure 1

Figure 1. Generation of an inducible Stat3 short-hairpin RNA mouse model. (a) Western blot of cell lysate from iMEF cells transfected with LMP retroviral vectors expressing candidate Stat3 targeting shRNAs. Data are representative of 2 independent experiments.

(b) Reporter gene assay showing 8XAPRE-Luc2 reporter signal as a measure of efficacy of the shRNA mediated Stat3 activity suppression. Data shows mean \pm SEM from technical triplicates.

(c) Schematic of the site-specific integration of the ColTGM vector that contains the shRNA into the genome of KH2 embryonic stem cells (ColA1 TRE-eGFP-miR-30). (1) A "landing pad" is knocked in on the 3'UTR site of the ColA1 locus of the KH2 embryonic stem cells (Beard et al., 2006). The landing pad consists of a PPGK and a neomycin resistant gene (NeoR) cassette that is flanked by frt sequences. Downstream to this cassette is an ATG-less hygromycin resistance cassette (HygroR). (2) The ColTGM vector comprises a strong Dox-responsive promoter (PTRE) to drive the expression of the eGFP and shRNA located downsteam to the promoter and ATG translational start codon, followed by an frt site to allow site specific recombination. (3) FLPe mediated recombination leads to excision of the PGK-NeoR cassette and site-specific recombination of the ColTGM vector in the ColA1 locus resulting in restoration of the hygromycin resistance.

(d) Western blot of four KH2 ES cell colonies targeted with the ColTGM Stat3.1348 plasmid and treated with or without Dox as indicated. Data are representative of 2 independent experiments.

(e) Representative IVIS derived images showing GFP expression in 2 weeks Dox treated shStat3 (left) and shStat3; rtTA (right) mice.

Figure 1

$247 \times 243 \mathrm{~mm}(300 \times 300 \mathrm{DPI})$

John Wiley \& Sons, Inc.

This article is protected by copyright. All rights reserved. 

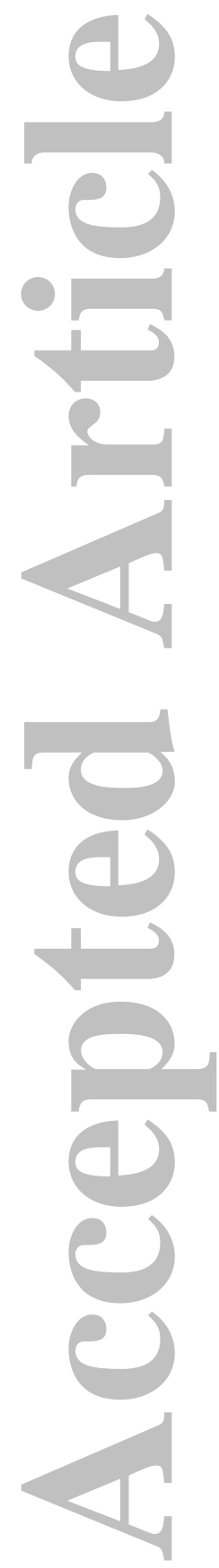

John Wiley \& Sons, Inc.

This article is protected by copyright. All rights reserved. 
a
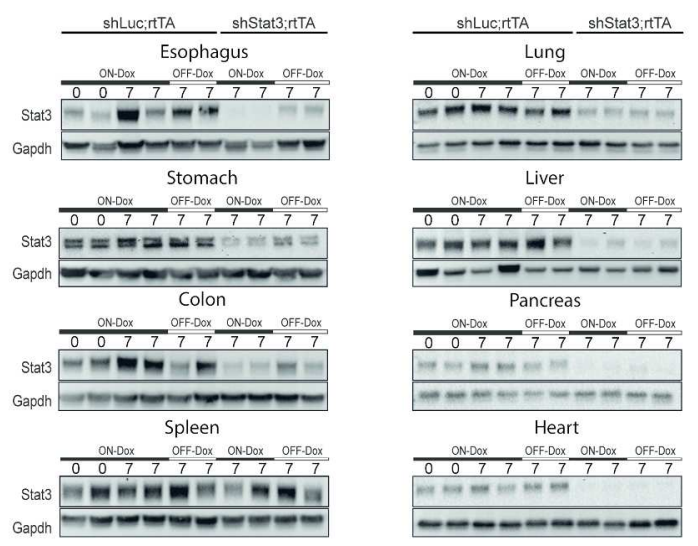

b
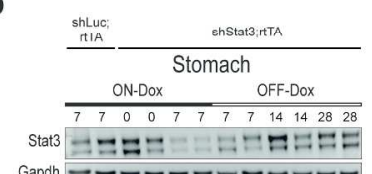

Gapdh ーーーーーーーーーーーー
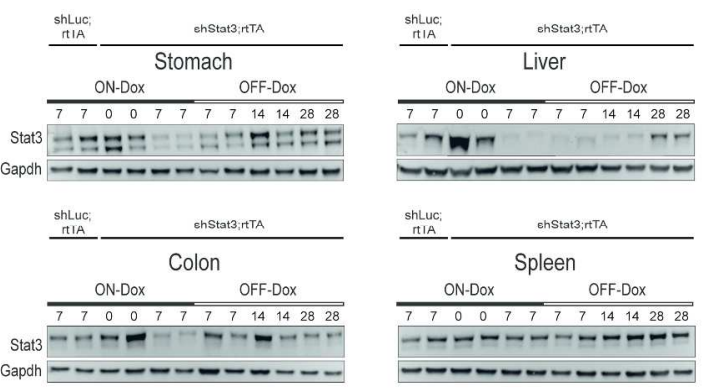

C

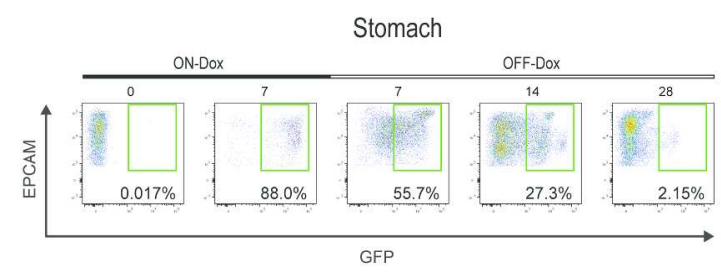

Figure 2

Figure 2. The level of shRNA-mediated Stat3 suppression and restoration is variable between different tissues.

(a) Western blot analysis of total Stat3 and Gapdh expression in whole tissue lysate from the indicated organs of shStat3;rtTA and control shLuc;rtTA mice, either left untreated or treated for 7 days with Dox and

analyzed directly after treatment (ON-Dox) or after 7 days of Dox withdrawal (OFF-Dox), one lane represents one mouse. Esophagus, pancreas, heart and lung westerns blots are representative of one experiment while stomach, liver colon and spleen blots are representative of 3 experiments.

(b, c) The shStat3; rtTA and control shLuc;rtTA mice were treated 7 days with Dox (ON-Dox) and were analyzed at $0,7,14$ or 28 days after Dox withdrawal (OFF-Dox).

(b) Western blot analysis of total Stat3 and Gapdh expression in whole tissue lysate from stomach, liver, colon and spleen of Dox-treated shStat3;rtTA and control shLuc;rtTA mice, each lane represents one mouse.

The time-points untreated, 7 days ON-Dox and 7 days OFF-Dox are representative of three independent experiments (each with $n=2-3$ mice), while the 14 days OFF-Dox are representative of 2 experiments (each

John Wiley \& Sons, Inc.

This article is protected by copyright. All rights reserved. 
with $n=2$ mice), and 28 days OFF-Dox are representative of one experiment ( $n=2$ mice). (c) Representative flow cytometric analysis of GFP expression on gated epithelial cells (Epcam+/CD45-) from enzymatically digested stomach tissue, each plot represents one mouse. Plots are representative of one experiment with 2 mice per time point.

Figure 2

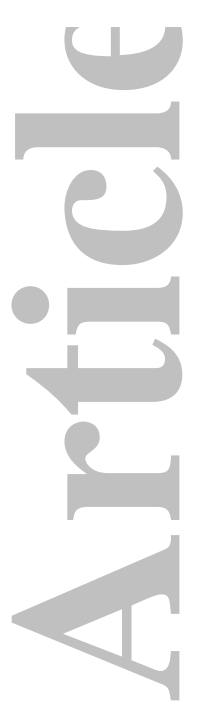

$172 \times 295 \mathrm{~mm}(300 \times 300$ DPI $)$

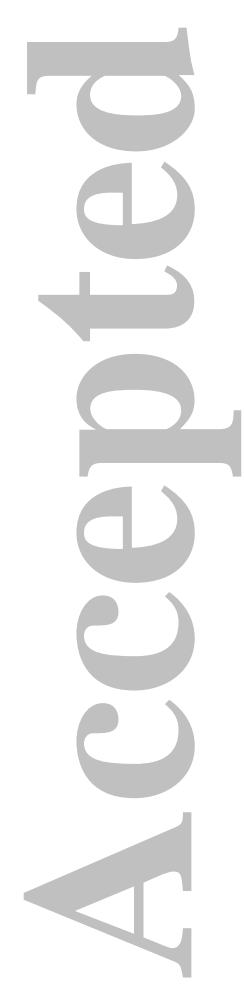

John Wiley \& Sons, Inc. 
a

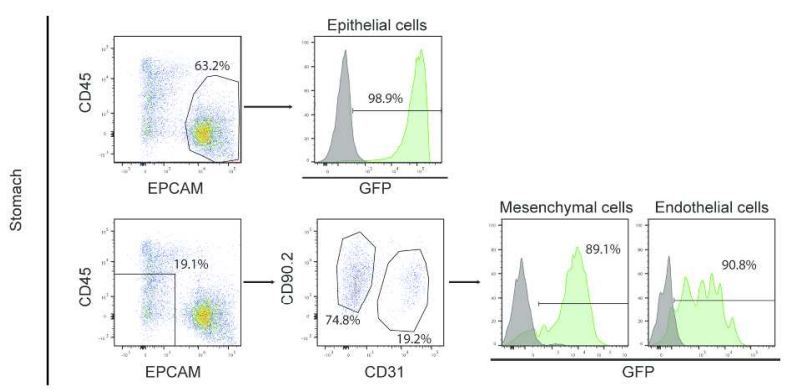

b
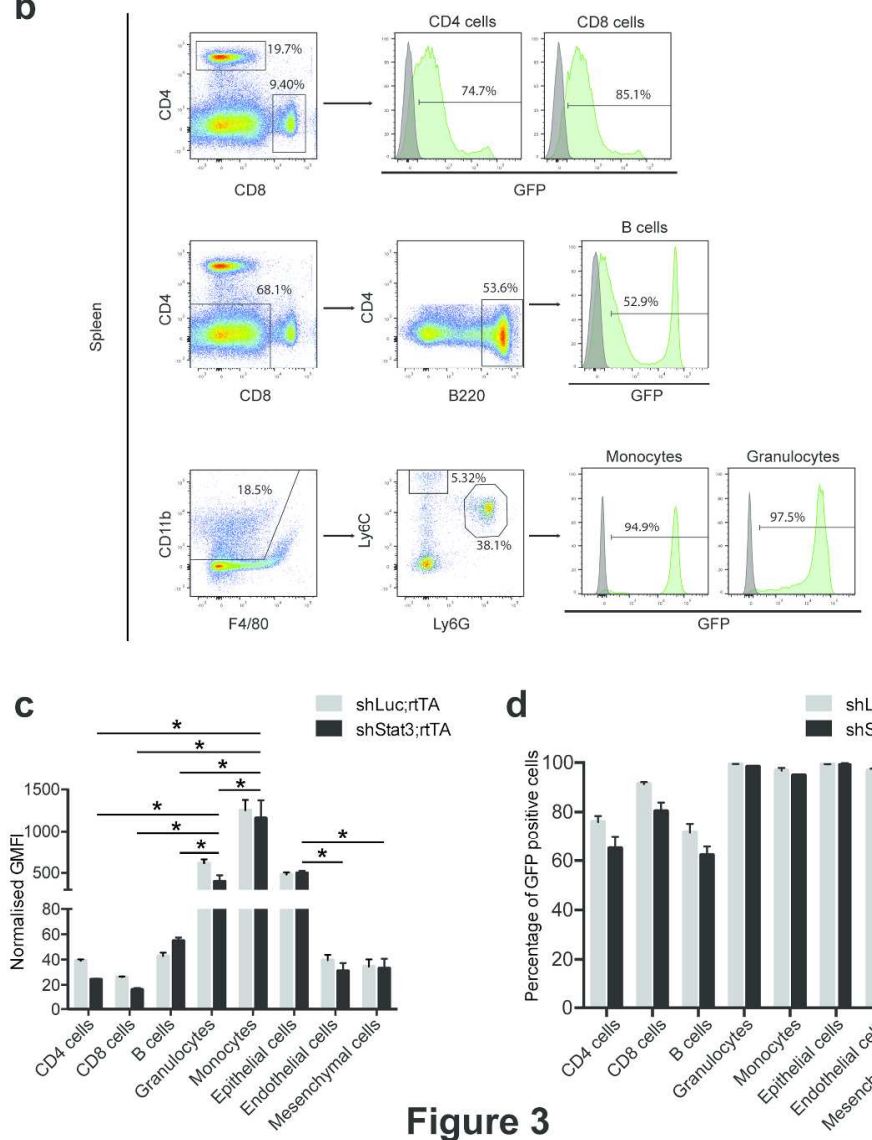

d
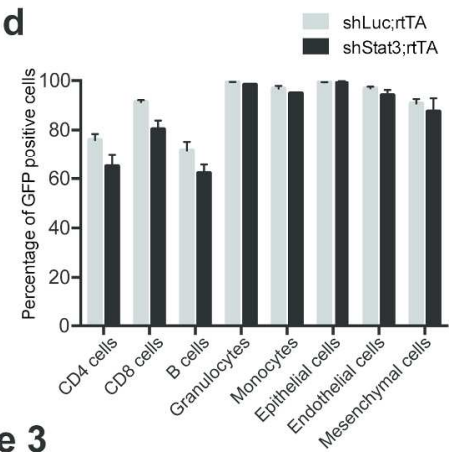

Figure 3. Heterogeneity of the shRNA expression across hematopoietic and non-hematopoietic cells. shStat3, shStat3;rtTA, and shLuc;rtTA mice were treated for 7 days with Dox.

(a) Representative FACS plots showing gating strategies and GFP expression in stomach epithelial (CD45/Epcam+), mesenchymal (CD45-/Epcam-/CD31-/CD90.2+) and endothelial cells (CD45-/Epcam/CD31+/CD90.2-) of shStat3;rtTA (green line) and shStat3 (grey line) mice, following 1 week of Dox treatment.

(b) Representative FACS plots showing gating strategies and GFP expression in the splenic CD4 T cells (CD4+/CD8-), CD8 T cells (CD4-/CD8+), B cells (CD4-/CD8-/B220+), monocytes (CD11b+/Ly6C+/Ly6G-) and granulocytes (CD11b+/Ly6Clow/Ly6G+) from shStat3;rtTA (green line) and shStat3 (grey line) mice following 1 week of Dox treatment.

(c) Bar graph shows the mean \pm SEM of the GFP geometric mean fluorescence intensity (GMFI) in shStat3;rtTA or shLuc;rtTA mice normalized to GFP GMFI in shStat3 mice within each of the indicated cell populations. Statistical differences were assessed with Mann-Whitney U statistical test. 
(d) Bar graph shows the mean \pm SEM percentage of GFP positive cells within each indicated population. Data are representative $(a, b)$ or cumulative $(c, d)$ of $n=4$ mice per genotype pooled from 2 independent experiments.

Figure 3

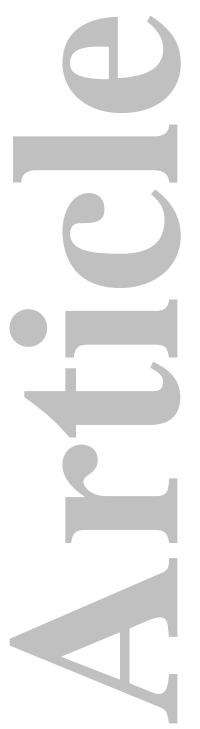

$191 \times 278 \mathrm{~mm}(300 \times 300$ DPI $)$

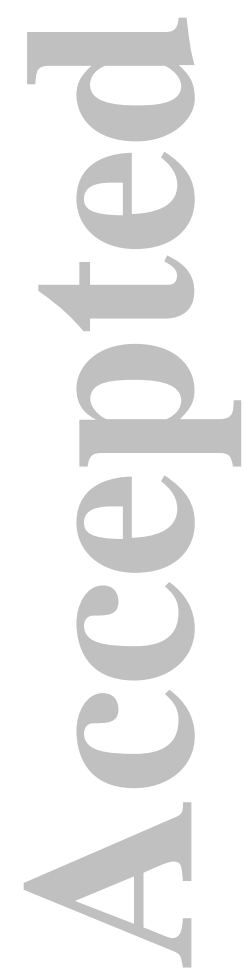

John Wiley \& Sons, Inc.

This article is protected by copyright. All rights reserved. 
a

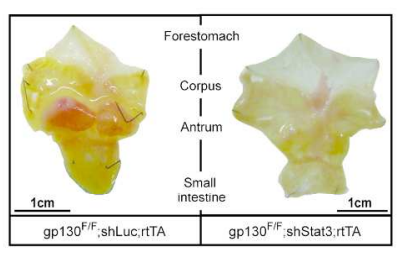

C

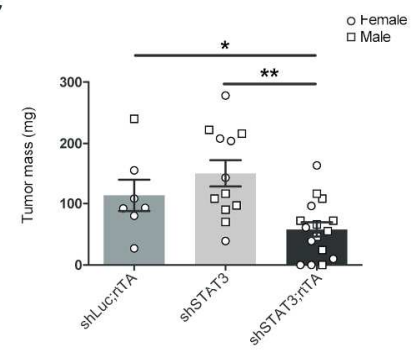

d

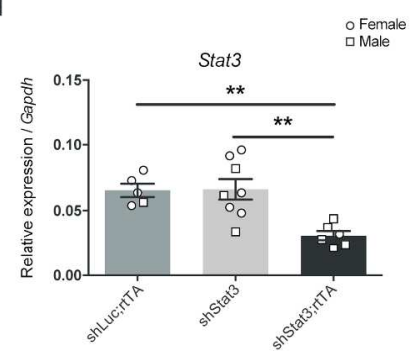

b

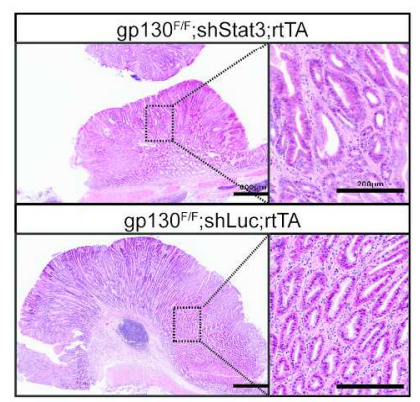

e

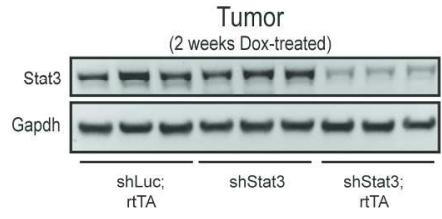

Figure 4

Figure 4. Stat3 silencing reduced tumor burden in a model of gastric tumorigenesis.

(a) Representative images of (18-20 weeks old) gp130F/F;shLuc;rtTAand gp130F/F;shStat3;rtTA mice after 2 weeks of Dox treatment.

(b) Hematoxylin \& Eosin-stained cross sections through stomachs from 2 weeks Dox-treated (18-20 weeks old) gp130F/F;shLuc;rtTA and gp130F/F;shStat3;rtTA mice. Images are representative of 6 gp130F/F; shLuc;rtTA mice from 4 independent experiments and $16 \mathrm{gp} 130 \mathrm{~F} / \mathrm{F}$; shStat3;rtTA mice from 5 independent experiments.

(c) Tumor mass in 2 weeks Dox-treated gp130F/F;shStat3;rtTA mice compared to gp130F/F; shLuc;rtTA and gp130F/F; shStat3 control mice, where one symbol represents one mouse. Data shows mean \pm SEM of the total tumor mass, with all shLuc;rtTA mice pooled from 3 independent experiments and shStat;rtTA and shStat3 mice pooled from 6 independent experiments.

(d) Quantitative RT-PCR analysis of Stat3 expression in gastric tumor tissue collected from 2 weeks Doxtreated gp130F/F;shStat3; rtTA compared to the gp130F/F;shLuc; rtTA and gp130F/F;shStat3 controls.

Values are normalized to Gapdh expression, and bar indicates mean \pm SEM of Stat3 normalized expression.

(e) Western blot analysis of total Stat3 and Gapdh in whole gastric tumor tissue of 2 weeks Dox-treated shStat3; rtTA. Samples are from 2 (shStat3), 3 (shLuc; rtTA), and 6 (shStat3; rtTA) independent experiments, each with $n=2-3$ mice.

The shLuc;rtTA mice are pooled from 3 independent experiments and the shStat3 and shStat3;rtTA are pooled from 6 independent experiments. Each symbol represents one mouse $(c, d)$. Statistical differences 
were assessed with Mann-Whitney U statistical test, $* \mathrm{p}<0.05 ; * * \mathrm{p}<0.01$. Figure 4

$212 \times 223 \mathrm{~mm}(300 \times 300 \mathrm{DPI})$
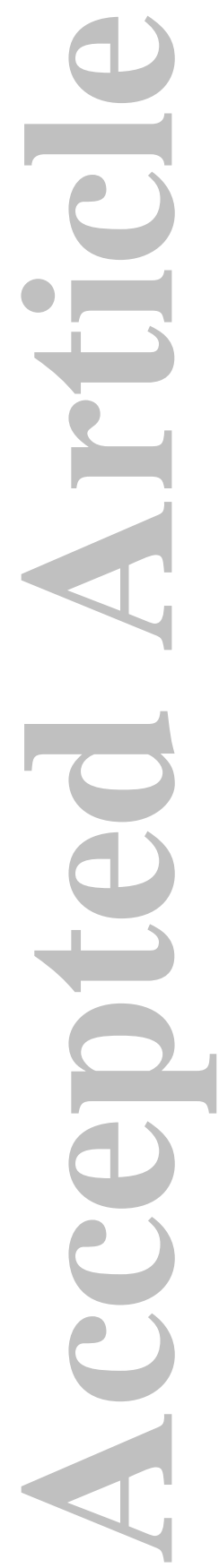

John Wiley \& Sons, Inc.

This article is protected by copyright. All rights reserved. 
a

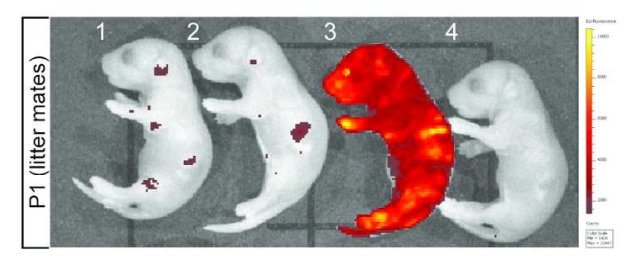

b

C

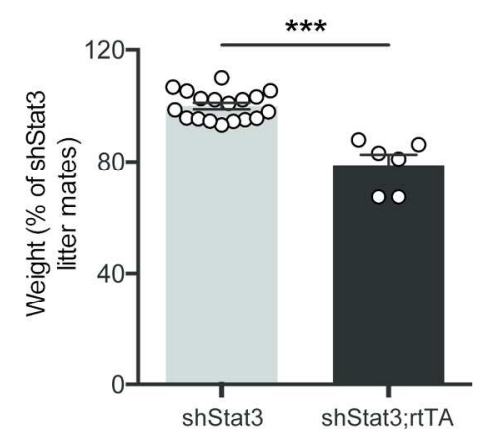

\section{Figure 5}

Figure 5. Stat3 inhibition during embryonic development results in decrease survival.

Wild-type females were mated with shStat3/shStat3;rtTA males and Dox was given throughout mating and pregnancy.

(a) Representative IVIS image showing GFP expression in 1 day old offspring.

(b) Table shows expected and observed number of offspring, and the Chi-square test value and its associated p-value, assessing statistical significant difference between observed and expected frequency of each genotype.

(c) Bar graph shows mean \pm SEM normalized total body weight of day 1-3 old offspring (normalized to the average body weight of shStat3 offspring) of shStat3;rtTA and shStat3 newborn mice where one symbol represents one mouse. Statistical differences were assessed with Mann-Whitney U statistical test.

Figure 5

$180 \times 181 \mathrm{~mm}(300 \times 300 \mathrm{DPI})$

John Wiley \& Sons, Inc.

This article is protected by copyright. All rights reserved. 


\section{University Library}

\section{- M M N E R VA A gateway to Melbourne's research publications}

Minerva Access is the Institutional Repository of The University of Melbourne

Author/s:

Alorro, MG;Pierce, TP;Eissmann, MF;Dijkstra, C;Dickins, RA;Ernst, M;Buchert, M;Masson, F

Title:

Generation of an inducible mouse model to reversibly silence Stat3

Date:

2017-04-01

Citation:

Alorro, M. G., Pierce, T. P., Eissmann, M. F., Dijkstra, C., Dickins, R. A., Ernst, M., Buchert, M. \& Masson, F. (2017). Generation of an inducible mouse model to reversibly silence Stat3. GENESIS, 55 (4), https://doi.org/10.1002/dvg.23023.

Persistent Link:

http://hdl.handle.net/11343/292476 